\title{
O USO DE UM SISTEMA DE AUTOMAÇÃO NA GESTÃO DE RECURSOS HÍDRICOS PARA A IRRIGAÇÃO PERIÓDICA DE HORTAS FAMILIARES: uma
}

\author{
proposta para uma casa de vegetação
}

THE USE OF AN AUTOMATION SYSTEM IN THE MANAGEMENT OF WATER

RESOURCES FOR THE PERIODIC IRRIGATION OF HOME GARDENS: a proposal

for a greenhouse

Carlos Alberto Schettini Pinto

CEFET - Rio de Janeiro - RJ - Brasil

DOI: 10.31510/infa.v18i1.1192

Data de submissão: 30/03/2021

Data do aceite: 09/07/2021

Data da publicação: 30/07/2021

\begin{abstract}
RESUMO
O presente trabalho apresenta o uso de uma rede de sensores sem fio, sendo controlados de forma centralizada ou descentralizada, em uma horta familiar. A proposta é a disponibilização de nós sensores que, a partir da obtenção de dados de umidade, temperatura e pressão e, utilizando dados pré-estabelecidos de cada tipo de hortaliça, possa irrigar de forma eficiente a plantação permitindo uma produção de qualidade, a custo baixo, mitigando a possibilidade de perdas no cultivo. A relevância deste estudo é comprovada a partir do momento em que possuímos, no Brasil, cerca de 5 milhões de pequenas propriedades rurais. A possibilidade desta forma de cultura familiar se inserirem no uso de uma tecnologia de vanguarda, por um custo baixo, lhes ofertando eficácia no resultado é bastante significativa. Em sua conclusão, o artigo destaca a acurácia do uso de nós sensores de forma centralizada ou descentralizada para o acionamento de irrigadores, proporcionado a economia no uso dos recursos hídricos.
\end{abstract}

Palavras-chave: Redes de sensores sem fio; casa de vegetação; descentralização de redes.

\begin{abstract}
The present work presents the use of a wireless sensor network, being controlled in a centralized or decentralized way, in a family garden. The proposal is to provide sensor nodes that, from obtaining data on humidity, temperature and pressure and, using pre-established data for each type of vegetable, can efficiently irrigate the plantation, allowing for quality production at cost low, mitigating the possibility of crop losses. The relevance of this study is proven from the moment that we have, in Brazil, about 5 million small rural properties. The possibility of this form of family culture being inserted in the use of cutting edge technology, at a low cost, offering them efficiency in the result is quite significant. In conclusion, the article highlights
\end{abstract}




\section{|WTERFFAET TECNOLÓGGCA}

the accuracy of the use of sensor nodes in a centralized or decentralized way to activate irrigators, providing savings in the use of water resources.

Keywords: Wireless sensor networks; Vegetation House; decentralization of networks.

\section{INTRODUÇÃO}

A agricultura familiar no Brasil possui forte impacto na economia nacional, sendo responsável por mais de 50\% da alimentação consumida no país, conforme nos diz (JACTO, 2019).

De acordo com o Censo Agropecuário que foi divulgado pelo Instituto Brasileiro de Geografia e Estatística (IBGE), em 2017, e citado por Barbosa (2020),

são 5 milhões de pequenas propriedades rurais em todo o país, representando $77 \%$ dos estabelecimentos da produção agrícola. No campo, a agricultura familiar foi responsável por R\$131,7 bilhões (23\%) dos R\$ 572,99 bilhões referentes ao Valor Bruto da Produção (VBP) brasileira naquele ano - a soma de tudo o que gira nas fazendas.

Em termos de empregos, são 10 milhões de postos de trabalho, com $67 \%$ do total da atividade agropecuária. (BARBOSA, 2020)

Corrobora para esta afirmação o constante no sítio do Ministério da Agricultura, Pecuária e Abastecimento (MAPA) onde afirma que a "Agricultura Familiar é a principal responsável pela produção dos alimentos que são disponibilizados para o consumo da população brasileira" (BRASIL, 2020a).

Destaca-se que este tipo de atividade permite a geração de empregos, principalmente na zona rural, fixando as famílias no campo, impedindo o êxodo para as grandes cidades, já bastante inchadas populacionalmente. Economicamente, dados do Censo Agropecuário 2017-2018 realizados pelo Instituto Brasileiro de Geografia e Estatística (IBGE) apresentados por estudos da Empresa Brasileira de Pesquisa Agropecuária (EMBRAPA), destacam que a agricultura familiar foi responsável por $23 \%$ do valor da produção agrícola brasileira e a agricultura nãofamiliar foi responsável por $77 \%$ desse valor. BRASIL (2017b).

Alguns agricultores familiares ainda realizam o seu cultivo a partir de metodologias bastante simples, preparando a terra, plantando as sementes, realizando irrigação manual, sem muitos controles na quantidade da água, além dos controles de luz, umidade, temperatura, etc. 
Com o advento da informatização dos meios, a possibilidade de se utilizar dispositivos inteligentes surgem como uma nova forma de se melhorar a qualidade na produção, atendendo às mais exigentes práticas produtivas, mesmo que sendo realizada de forma familiar ou por pequenos produtores. O uso destes dispositivos é importante pois tem a capacidade de medirem "on time" uma série de parâmetros e decidirem, a partir de respostas pré-determinadas, quais as ações a serem realizadas, sem a necessária interferência humana. Trstenjak e Cosic, (2017), citam que os princípios da Indústria 4.0 exigem uma mudança geral por digitalização e automatização de todas as partes da empresa, junto com o processo de fabricação. Pode-se trazer este entendimento para o conceito agroindustrial, ao automatizarmos os processos de controle de irrigação, do plantio, e, até mesmo da colheita. Surgindo como um dos recursos fundamentais, a automação hídrica se apresenta como um novo mercado com potenciais ainda em desenvolvimento, colaborando na gestão hídrica e no manejo de processos que demandem recursos hídricos nos processos, gerando economia e precisão na manutenção das condições ideais ao cultivo.

Loureiro et al (2003) citam o uso de Redes Sensores Sem Fio (RSSF):

Uma RSSF pode ser usada para monitorar e, eventualmente, controlar um ambiente. Este tipo de rede é formado geralmente por centenas ou milhares de dispositivos autônomos que tendem a ser projetados com pequenas dimensões $\left(\mathrm{cm}^{3}\right.$ ou $\left.\mathrm{mm}^{3}\right)$ chamados nós sensores. Os principais componentes de um nó sensor são transceptor para comunicação sem fio, fonte de energia, unidade de sensoriamento, memória e processador. O componente lógico de um nó sensor é o software que executa no processador (LOUREIRO et al, 2003).

Não obstante, é importante delinear dois entendimentos que serão fundamentais para o estudo: A centralização e a descentralização das redes de sensores sem fio. Trabalhando de forma colimada com a Internet das Coisas (IoT), os comandos poderão ser acionados de forma centralizada ou descentralizada, dependendo da forma com que o mesmo foi idealizado.

De acordo com a empresa CISCO(s.d), a implantação de uma rede centralizada,

é o tipo mais comum de sistema de rede sem fio. As implantações centralizadas são tradicionalmente usadas nos campus, onde os edifícios e as redes ficam próximos. Essa implantação consolida a rede sem fio, facilitando as atualizações e habilitando funcionalidades sem fio avançadas. Os controladores são locais e instalados centralmente. (CISCO, s.d.)

No que concerne a rede descentralizada, o Ispblog (2016) menciona que 
Uma Rede Descentralizada nada mais é do que uma rede onde as tarefas e os serviços não são centralizados. Ou seja, é uma rede indicada quando não há a necessidade de centralização do gerenciamento de tráfego e recursos. (ISPBLOG, 2016).

A seguir será apresentada uma perspectiva do uso da IoT num ambiente de agricultura familiar, de forma descentralizada.

\section{FUNDAMENTAÇÃO TEÓRICA}

No referencial teórico serão apresentados os seguintes tópicos: agricultura familiar e casa de vegetação, centralização e descentralização de redes sem fio.

\subsection{Agricultura Familiar e Casa de Vegetação}

Segundo Alves (2011), a casa de vegetação é uma estrutura coberta com materiais transparentes que serve de proteção contra agentes meteorológicos, tais como: granizo, excesso de chuvas, proteção do solo contra lixiviação, radiação solar acentuada e quedas bruscas de temperatura. Desta forma, o seu interior favorece o desenvolvimento de culturas durante seu ciclo vegetativo.

Para Silva (2019), a coleta de uma grande quantidade de dados passa a ser parte das técnicas de manejo e segue destacando que os exemplos de dados coletados incluem medições de temperatura, umidade, condutividade elétrica do solo e de nível de produtividade durante a colheita, etc.

\subsection{Redes Sem Fio Centralizadas}

Neste tipo de rede todos os nós se conectam a um servidor central que analisa os dados e toma sua decisão a partir destas análises.

Uluski (2010) descreve sobre a estratégia centralizada onde se tem um servidor e todos os equipamentos dedicado à regulação e à compensação de nós reativos que se comunicam com uma central, a qual define a operação de cada equipamento, com base nas medições que os próprios equipamentos retornam. E continua, mencionando que a estratégia centralizada de controle integrado, permite a função de redução de tensão com o objetivo de redução da demanda de consumo de energia no sistema de energia.

Fang et al., (2018) chama a atenção para o fato de que em redes sem fio que possuem uma entidade de controle centralizada capaz de remotamente controlar o acionamento e desligamento das interfaces de comunicação sem fio, pode-se utilizar um modelo capaz de realizar previsões para a presença ou não de associações entre alguma estação e um ponto de 


\section{WTEERFACETECNOLOGGCA}

acesso da rede, durante uma faixa de tempo, para então executar essas ações e economizar energia sem impactar a capacidade e cobertura da rede.

$\mathrm{Na}$ figura 1, percebe-se os nós sensores funcionando em conjunto e, ao informarem os valores de suas medidas ao computador central/controlador, este, de forma centralizada e utilizando os parâmetros existentes, decidirá qual tipo de acionamento será realizado. Desta forma, ele decidirá o acionamento, de um ou mais, dos módulos existentes. Como o sistema continua monitorando o ambiente, novas informações serão recebidas e o computador posteriormente, comandará o encerramento da hidratação por haver chegado aos padrões prédeterminados.

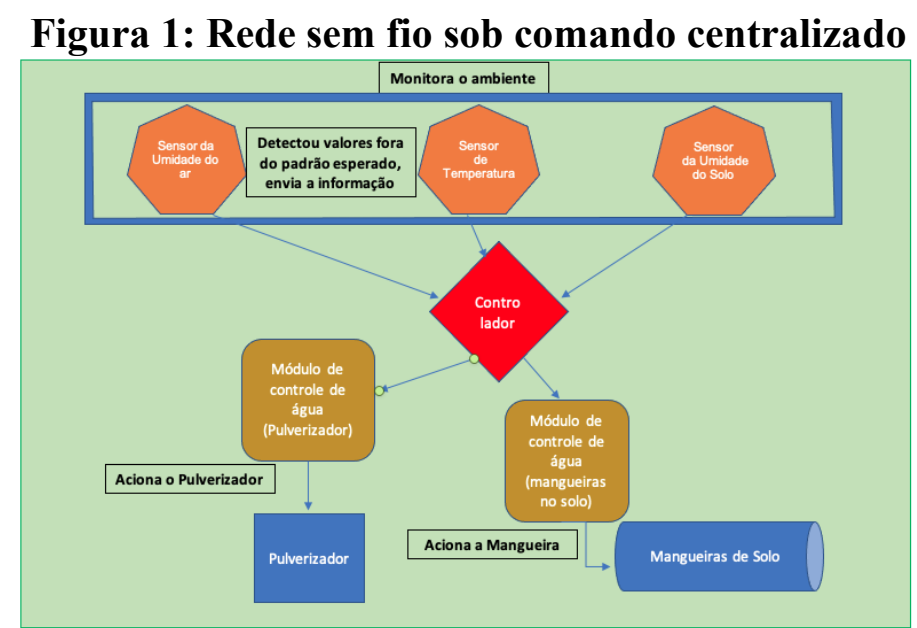

\section{Redes Sem Fio Descentralizadas}

Fonte: autor (2021)

Uma rede sem fio descentralizada é aquela onde as ações não ocorrem de forma centralizada, não necessitando que todos os dispositivos estejam conectados, agindo de forma independente a partir da constatação de que algum parâmetro que este esteja controlando necessite ser acionado ou comandado.

Loureiro et al., (2003) afirmam que "existem casos em que uma RSSF também pode ser composta de dispositivos chamados atuadores que permitem ao sistema controlar parâmetros do ambiente monitorado". Esta definição corrobora com o entendimento das redes descentralizadas cujos nós sensores possuem capacidade de controlar as ações sem a necessidade de controladores centralizados, permitindo agilidade no processamento de dados e fluidez no comandamento das ações.

Müller et al., (2013) propõem a utilização de coprocessadores, que são dispositivos com um nível de inteligência maior do que os dispositivos comuns, e que por isso, permitem o 
gerenciamento descentralizado. O coprocessador auxilia durante o evento de agregação de dispositivos intermitentes modificando o escalonamento das mensagens dinamicamente.

Segundo Cainelli (2020), estudos de casos mostram que o gerenciamento local resulta em reduções de tempos de agregação, uma vez que o processo de escalonamento é transferido do gerenciador central para o coprocessador.

Lieira et al., (2018) afirmam a importância de uma arquitetura de comunicação descentralizada e flexível, a qual permita o gerenciamento de mobilidade de múltiplas interfaces de redes ativas de forma simultânea, considerando tanto pontos de acesso com a mesma tecnologia de redes, como tecnologias diferentes.

Para Bang et al., (2013), as redes sem fio em que não existe um ponto de acesso e nas quais os dispositivos se comunicam diretamente entre si são conhecidas como redes móveis $a d$ hoc (MANETs) e tornaram-se uma importante tecnologia emergente em computação móvel, sendo uma das áreas de pesquisa mais prevalentes nos últimos anos.

\section{PROCEDIMENTOS METODOLÓGICOS}

O presente artigo utiliza-se da metodologia da pesquisa bibliográfica no qual buscou-se realizar um estudo a partir da utilização de redes sensores sem fio em um sistema de agricultura familiar. A revisão bibliográfica insere-se num contexto de revisão integrativa à medida que permite ao pesquisador cotejar a problemática que deseja apreciar e, ao mesmo tempo, autoriza-o a traçar um panorama lógico em todo o desenvolver da produção científica. Para o artigo em questão, foram utilizadas as principais bases de dados disponíveis, além da consulta a sítios organizacionais aderentes aos assuntos discutidos.

\section{DISCUSSÃO}

\subsection{O Processo Automatizado}

Com o "nós sensores" serão utilizados o higrômetro para medir a umidade do solo; o sensor de umidade, para medir a umidade do ambiente; um sensor de temperatura para mensurar a temperatura ambiental; e, um medidor de pressão atmosférica.

Para Ray (2016), os nós sensores (i.e., dispositivos de comunicação munidos de sensores) são parte integrante da Agricultura de Precisão, sendo responsáveis por realizar o monitoramento, medições e a coleta de dados. 


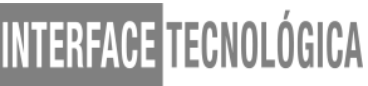

Segundo Majone et al., (2013), os nós sensores são elementos fundamentais de um sistema de IoT e a precisão nas medições é uma característica determinante das diversas aplicações, considerando que a calibragem executada nesses componentes reflete a acurácia dos resultados.

Estes nós serão estacionários, isto é, ficarão sempre em uma mesma posição sendo totalmente automatizados de forma que os nós atuadores coordenam-se entre si e decidem um plano de ação baseando-se nos dados detectados e nas informações previamente disponibilizadas.

Karimi (2013) ressalta que nos ambientes de IoT, os dados costumam ser trocados entre dispositivos e trafegados nas redes de comunicação sem fio de forma que sejam processados localmente por MCUs (Microcontroller Unit) ou MPUs (Microprocessor Unit), ou enviados para servidores centralizados ou aplicações baseadas em nuvem.

Abordando a topologia a ser empregada, poderia ser utilizada a estrela ou malha. Ambas estão sujeitas a falhas nos nós, principalmente se as condições ambientais não forem favoráveis e os nós sofrerem constantes mudanças de localização.

Na topologia estrela, todos os nós se comunicam com uma estação base, não havendo nenhum tipo de comunicação não intermediada por ela. Neste tipo de topologia, a área de cobertura fica limitada ao alcance de comunicação da estação base. De acordo com Rawat et $a l .$, (2014), a topologia em estrela é preferida quando a área de cobertura é pequena e baixa latência é requerida pela aplicação.

$\mathrm{Na}$ topologia em malha, segundo Santos (2019), os nós atuam como roteadores, encaminhando pacotes de outros nós da rede e a sua comunicação ocorre entre os nós e o gateway (que conecta à rede externa) ou entre os próprios nós. Neste modo de operação, obstáculos que impeçam a comunicação podem ser contornados através dos nós intermediários, ampliando-se assim a confiabilidade da rede. Múltiplos caminhos podem ser construídos dinamicamente para levar os dados de uma extremidade à outra da rede e a área de cobertura pode ser ampliada.

Como dito anteriormente, ambas as topologias poderiam ser utilizadas, mas propõemse a topologia em malha, para o caso de ocorrência de obstáculos que possam vir a impedir o tráfego correto das informações. Além disso, a utilização de redundância de nós-sensores, lendo a mesma informação, pode gerar uma maior acurácia na leitura dos dados. 
A figura 2 apresenta uma visão holística de todo o processo de funcionamento do RSSF. Como percebe-se na imagem, o ambiente sofre a ação do sol, chuva, ventos, etc. Os sensores realizarão o monitoramento "on time" dos parâmetros previamente pré-estabelecidos. Uma vez que ocorra uma discrepância, como por exemplo, solo seco, aumento da temperatura ambiente, etc. Ele emitirá um sinal sem fio para o módulo de controle de água. Este, acionará aos pulverizadores de água que espargirão determinada quantidade de água permitindo que o ambiente volte a ficar úmido dentro dos padrões exigidos, ou mesmo que o solo volte a ficar propício ao desenvolvimento e à manutenção do status quo para aquela hortaliça. Da mesma forma, caso o terreno se encontre seco, isto é, fora dos valores padrão de umidade desolo para aquele tipo de hortaliça, o sensor emitirá um comando para o acionamento das mangueiras de hidratação do solo. Este é um procedimento dinâmico e constante, onde a ação humana não se faz tão premente, mas que permite um acompanhamento dos procedimentos realizados, das variáveis de pressão, temperatura e umidade, dentre outros parâmetros que o cultivador julgue ser úteis para o correto acompanhamento e desenvolvimento de seus produtos.

Há a possibilidade de serem anexados outros sensores com outras informações que os responsáveis pelo cultivo de determinado insumo julguem pertinentes.

Figura 2: Processo de funcionamento do RSSF

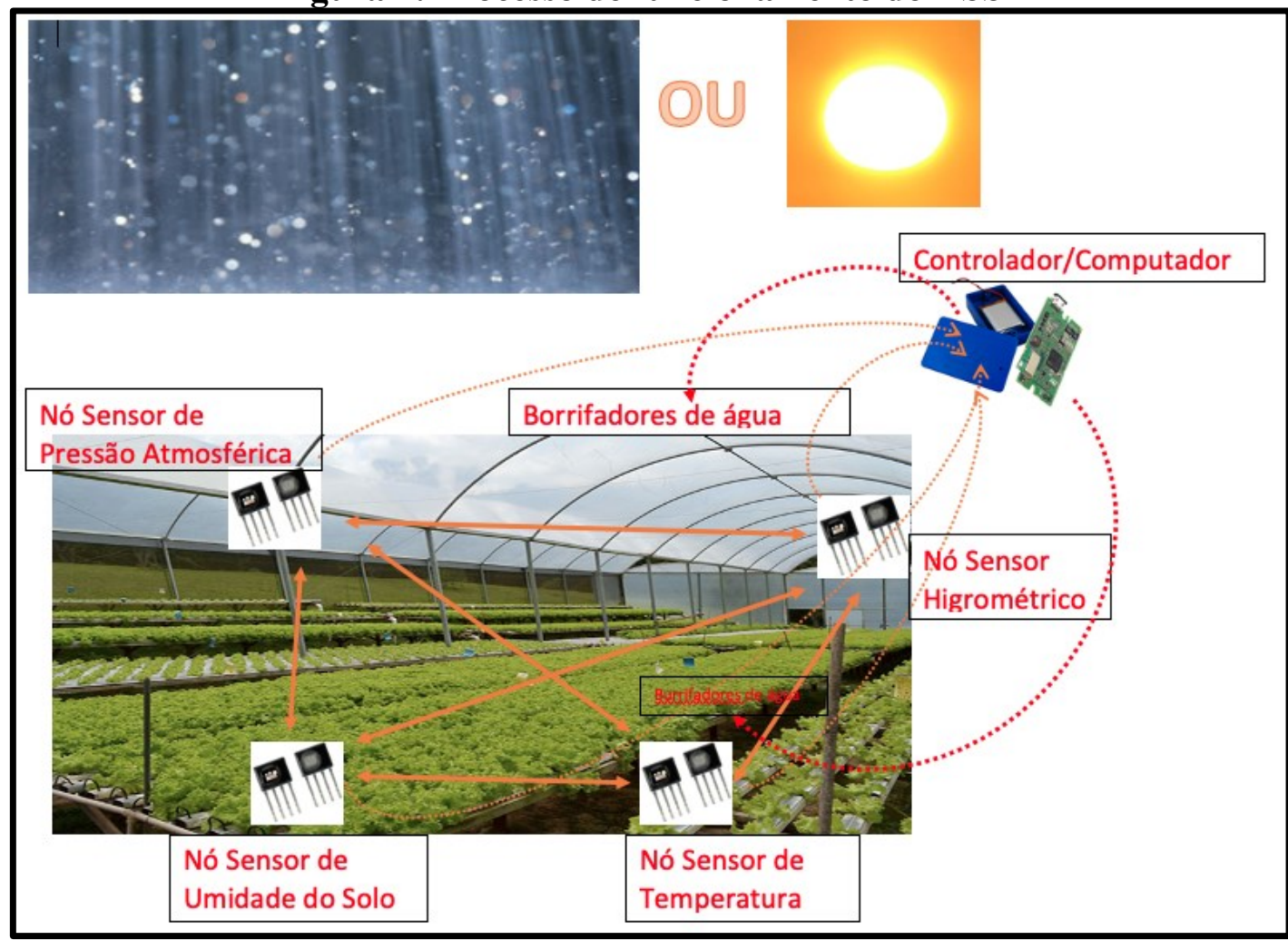

Fonte: autor 


\section{NTIEREACETECNOLOGGCA}

Os nós-sensores poderão ser unitários por tipo de sensoriamento medido ou serem redundantes, colocando-os em posições distintas realizando medidas em outros pontos, o que garante uma acurácia maior na obtenção dos dados. Outro fator a ser considerado é que, havendo mais de um tipo de hortaliça, os valores parametrizados de cada insumo podem apresentar diferenças e, o nó-sensor deve observar estas variâncias. Para tanto é importante, ao se estabelecer os critérios de cada nó-sensor, que o mesmo atenda aos padrões daquela hortaliça a qual ele encontra-se monitorando.

Na Figura 3, a visão esquematizada da ação dos nós-sensores/controladores que foi explicada anteriormente.

\section{Figura 3: Esquema de funcionamento descentralizado dos nós sensores}

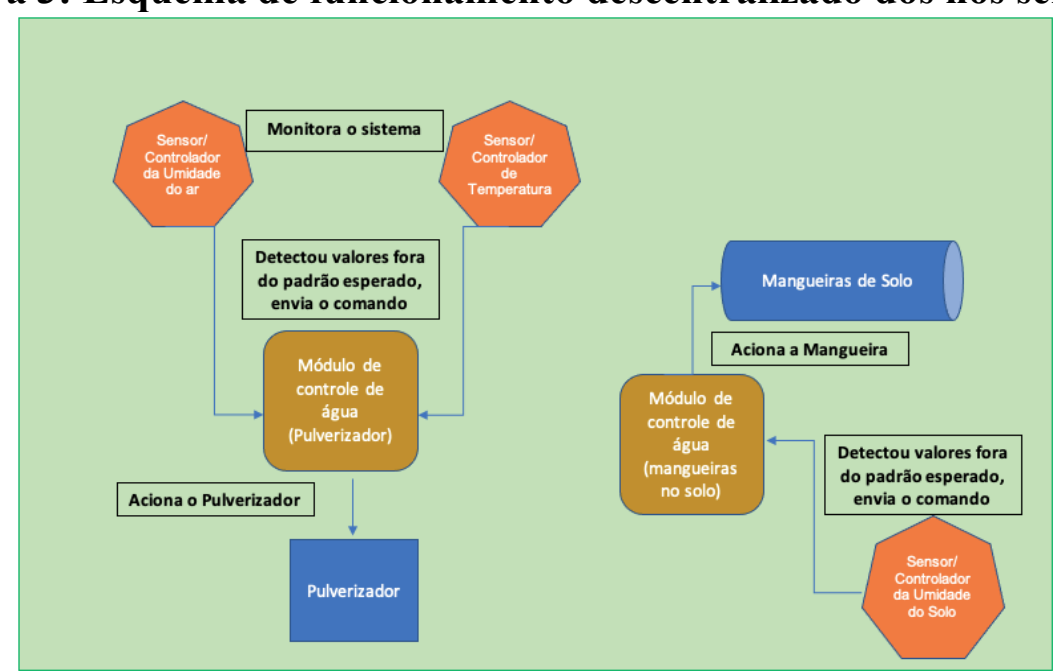

Fonte: autor (2021).

Cabe ainda ressaltar que os controles de quantidade de água no solo, sua umidade e temperatura também ajudam no combate aos fungos e bactérias. As hortaliças se caracterizam pelo elevado teor de água em seus tecidos, favorecendo ao ataque de doenças de plantas. Flutuações no potencial de água no solo afetam sobremaneira a atividade metabólica, o crescimento vegetativo e a reprodução de vários microrganismos de solo, inclusive os fitopatogênicos, resultando em maior ou menor intensidade, manutenção e propagação de uma doença. Um estudo da EMBRAPA (1996,d) chama a atenção citando

Os maiores problemas da água com relação às hortaliças advêm, entretanto, do seu excesso associado a altas temperaturas, situação frequente em regiões de países tropicais como o Brasil. Sob estas condições as doenças se propagam mais fácil e intensamente, e limitam o cultivo de várias hortaliças, como as solanáceas e algumas raízes e tubérculos. (BRASIL, 1996,d) 


\subsection{Parâmetros de Medidas}

De acordo com os dados da EMBRAPA, de modo geral, as hortaliças encontram melhores condições de desenvolvimento em climas amenos, com chuvas leves e pouco frequentes. Baixas temperaturas retardam o crescimento, a frutificação e a maturação. Temperaturas mais altas são adequadas na época do florescimento e da maturação. Cada hortaliça tem suas exigências climáticas, mas pode-se dizer que, de modo geral, as folhosas e as raízes se desenvolvem melhor entre 15 e $23^{\circ} \mathrm{C}$, enquanto as hortaliças-frutos produzem mais entre 18 e $25^{\circ} \mathrm{C}$ (algumas hortaliças desse tipo sobrevivem em temperaturas na faixa de 30 a $35^{\circ} \mathrm{C}$, mas a frutificação é bastante prejudicada). Couve e repolho suportam temperaturas mais baixas e até geadas leves (BRASIL, 1993c, p. 12-13).

Para fins de estudos futuros e testagem em softwares de simulação, sugere-se utilizar as seguintes hortaliças, irrigação e suas temperaturas-alvo, constantes da Tabela 1:

Tabela 1: Hortaliças com seus parâmetros (adaptado)

\begin{tabular}{c|c|c}
\hline Hortaliça & Irrigação & Parâmetros de Temperatura $^{\mathbf{0}} \mathbf{C}$ \\
\hline Abóbora e Moranga & $\begin{array}{c}\text { Diariamente por 30 dias após o } \\
\text { plantio e de 4 em 4 dias até o } \\
\text { início da maturação }\end{array}$ & 20 a $27^{\circ}$ \\
\hline Alface e Chicória & $\begin{array}{c}\text { Durante Semente, duas vezes ao } \\
\text { dia. Diariamente após a eclosão. }\end{array}$ & 12 a $22^{\circ}$ \\
\hline Batata-Doce & De 3 em 3 dias & 16 a $23^{\circ}$ \\
\hline Pimenta & $\begin{array}{c}\text { Durante semente, diariamente. } \\
\text { Após eclosão em intervalos de 3 } \\
\text { a 4 dias. }\end{array}$ & 18 a $25^{\circ}$ \\
\hline Tomate & $\begin{array}{c}\text { Durante semente, diariamente. } \\
\text { Após eclosão em intervalos de 3 } \\
\text { a 4 dias. }\end{array}$ & 15 a $25^{\circ}$ \\
\hline
\end{tabular}

Fonte: BRASIL, 1993c

\section{CONCLUSÃO}

O presente estudo buscou verificar a situação de uma agricultura familiar a partir da utilização da casa de vegetação e apresentar uma possibilidade de utilização da mesma a partir da incorporação de dispositivos provenientes da IoT, demonstrando que, mesmo em ambientes mais simples, é possível a adoção de dinâmicas tecnológicas para auxiliar nas atividades agrícolas.

A área das redes sem fio tem sido tema constante de estudo e inovação tecnológica. É mister compreender que o sensoriamento remoto não é mais “o futuro", mas sim, o presente, ocorrendo 
em constantes atualizações e desenvolvimentos para os ambientes industriais, corporativos, residenciais, agropecuário, militar, medicinal, etc.

Em ambientes tecnológicos onde as partes se comunicam, é altamente desejável que os sistemas possuam inter-relacionamento permitindo a troca de conhecimento, alimentando repositórios com informações relevantes que possam ser utilizadas para diversos fins, como pesquisa, desenvolvimento, estudos prospectivos, etc.

Numa visão prospectiva, os dados colhidos no ambiente de estudo poderiam ser compartilhados com outros produtores, de forma colaborativa, combinando os dados coletados proporcionando dados robustos e permitindo a realização de métricas que gerariam previsões meteorológicas, por exemplo, ou mesmo beneficiando outros projetos visando redução de gastos com água, energia, danos ao meio ambiente, etc.

Verificou-se que o uso de sensores pode ser bastante interessante nesses ambientes por proporcionarem um controle mais acurado da parte hídrica, proporcionando menor desperdício, permitindo que as hortaliças possam estar sempre dentro dos parâmetros exigidos de temperatura, umidade e pressão, resultando em produtos melhores.

Não obstante, os controles poderiam atuar de forma centralizada ou descentralizada, sendo que esta última maneira, permitiria uma dinâmica maior dos nós-sensores, atuando como controladores, diminuindo os números de faseamentos, gerando agilidade e reduzindo a necessidade de equipamentos. Considerando uma agricultura de baixo valor agregado, qualquer tipo de redução de custos é um fator de ganho de escala a ser considerado favoravelmente na escolha desta forma de controle.

Por fim, o presente estudo ressalta a possibilidade da adoção desta sistemática, devendo ser aprofundado na escolha dos tipos de sensores, buscando aquele que melhor atenda a determinada demanda; na possibilidade de escolher os tipos de hortaliças e seus parâmetros de valores agregados; e, principalmente, compreender que a IoT pode ser percebida como uma grande vantagem competitiva, mesmo para pequenos produtores, em virtude da sistematização e acurácia no controle e gestão das ações a serem executadas nas hortas familiares ou de pequeno porte.

\section{REFERÊNCIAS}

ALVES, E. C. de S. Avaliação de Parâmetros de Desempenho de Rede de Sensores Sem Fio em Casa de Vegetação para o Cultivo Hidropônico de Morangos. 2011. Dissertação de 
Mestrado. UNICAMP. Campinas - SP Disponível em:

http://repositorio.unicamp.br/jspui/handle/REPOSIP/256853?mode=full. Acesso em 03/02/2021.

BANG, O.A.; RAMTEKE, P. MANET: History, Challenges and Applications.

International Journal of Application or Innovation in Engineering \& Management, v. 2, Issue 9, Sep- tember 2013.

BARBOSA, F. Agricultura familiar emprega mais de 10 milhões de pessoas no Brasil.

Revista Globo Rural. 25 jul 2020. Disponível em:

https://revistagloborural.globo.com/Noticias/Agricultura/noticia/2020/07/agricultura-familiaremprega-10-milhoes-de-pessoas-no-brasil.html. Acesso em 12/02/2021.

BRASIL.(a) Ministério da Agricultura, Pecuária e Abastecimento. Agricultura Familiar. 04/05/2020. Disponível em: https://www.gov.br/agricultura/pt-br/assuntos/agriculturafamiliar/agricultura-familiar-1. Acesso em 12/02/2021.

.(b) Embrapa. Artigo - Qual é a participação da agricultura familiar na produção de alimentos no Brasil e em Rondônia?. 08/09/2020. Disponível em:

https://www.embrapa.br/busca-de-noticias/-/noticia/55609579/artigo---qual-e-a-participacao-daagricultura-familiar-na-producao-de-alimentos-no-brasil-e-em-rondonia. Acesso em 23/05/2021.

DF. 1993.

.(c) Embrapa. O Cultivo de hortaliças. Serviço de Produção de Informação. Brasília -

(d) Embrapa. Manejo da irrigação em Hortaliças. Serviço de Produção de

Informação. Brasília - DF. $5^{\text {a }}$ Edição. 1996.

CAINELLI, G. P. Proposta de algoritmo de escalonamento de mensagens para redes sem fio aplicadas à automação de fábrica. Dissertação de Mestrado em Engenharia Elétrica da UFRGS. 2020. Disponível em:

https://www.lume.ufrgs.br/bitstream/handle/10183/216443/001120101.pdf?sequence=1\&isAl lowed $=$ y. Acesso em 22/02/2021.

CISCO. O que é uma rede sem fio? Disponível em:

https:/www.cisco.com/c/pt_br/solutions/small-business/resource-center/networking/wirelessnetwork.html. Acesso em 06/06/2021.

FANG, L., XUE, G., LYU, F., SHENG, H., ZOU, F., LI, M. (2018). Intelligent large-scale ap control with remarkable energy saving in campus wifi system. In 2018 IEEE $24^{\text {th }}$ International Conference on Parallel and Distributed Systems (ICPADS), pages 69-76.IEEE.

ISPBLOG. Rede descentralizada: entenda em que consiste este modelo de rede. 26/09/2016. Disponível em: https://www.ispblog.com.br/2016/09/26/rede-descentralizada-entenda-emque-consiste-esse-modelo-de-rede/. Acesso em 02/02/2021.

JACTO. Agricultura familiar no Brasil: importância para a economia do país. 18/09/2019. Disponível em: https://blog.jacto.com.br/agricultura-familiar-no-brasil/. Acesso em 12/02/2021.

LIEIRA, D. D.; VALENTINI, E. P.; NAKAMURA, L. H. V.; MENEGUETTE, R. I. Uma Nova Arquitetura Descentralizada para Mobilidade de Fluxo em Redes Veiculares. In: WORKSHOP DE GERÊNCIA E OPERAÇÃO DE REDES E SERVIÇOS (WGRS), 23, 2018, Campos do Jordão. Anais[.]. Porto Alegre: Sociedade Brasileira de Computação, 2018 . 
LOUREIRO, A. A. F; NOGUEIRA, J. M. S; RUIZ, L. B; MINI, R. A. de F; NAKAMURA, E. F; FIGUEIREDO, C. M. S. Redes de Sensores Sem Fio. Anais do XXI Simpósio Brasileiro de Redes de Computadores (SBRC'2003), Natal, 19 a 23 de Maio de 2003.

MAJONE, B., VIANI, F., FILIPPI, E., BELLIN, A., MASSA, A., TOLLER, G., ROBOL, F., SALUCCI, M. Wireless Sensor Network Deployment for Monitoring Soil Moisture Dynamics at the Field Scale. Procedia Environmental Sciences 19. 2013, 426-435.

MÜLLER, I. et al. WirelessHART Fast Collect: a decentralized approach for intermittent field devices. In: IEEE INTERNATIONAL CONFERENCE ON INDUSTRIAL

INFORMATICS (INDIN), 11., 2013. Proceedings [... [S.1.: s.n.], 2013. p. 254-259.

KARIMI, K. What the Internet of Things (IoT) needs to become a reality. White Paper, FreeScale and ARM (2013).

RAWAT, P., SINGH, K. D., CHAOUCHI, H., BONNIN, J. M. Wireless sensor networks: A survey on recent developments and potential synergies. Journal of Supercomputing 68, 1 (2014), 1-48.

RAY, P. A survey on Internet of Things architectures. Journal of King Saud University Computer and Information Sciences, 2016.

TRSTENJAK, M. e COSIC, P. "Process planning in Industry 4.0 environment", Procedia Manufacturing, vol. 11, junho, pp. 1744-1750, 2017.

ULUSKI, R. "VVC in the smart grid era," IEEE Power and Energy Society General Meeting, 2010. 